\title{
O PSICÓLOGO, A SAÚDE PÚBLICA E O ESFORÇO PREVENTIVO*
}

\author{
Nilce Pinheiro Mejias**
}

\begin{abstract}
MEJIAS, N. P. O psicólogo, a saúde pública e o esforço preventivo. Rev. Saúde públ., S. Paulo, 18: 155 - 61, 1984.

RESUMO: Foi levada a efeito revisão de vários artigos concernentes aos problemas da influência do comportamento do indivíduo na manutenção de sua saúde e das relaçōes entre aspectos do ambiente, comportamento e saúde. Considerando os pontos de contacto e de divergência entre psicologia clínica e saúde pública, sugere-se que a psicologia da comunidade é a área da psicologia cujos objetivos mais se aproximam aos da Organizaçz̃o Mundial de Saúde - uma sugestão ditada por suas preocupaçōes comuns com as relaçōes entre os sistemas de organizaça social e o funcionamento individual e com o desenvolvimento de sistemas sociais condizentes com as necessidades humanas. Tendo em vista a escassez de dados empíricos, conclui-se que, para uma maior contribuição da psicologia à saúde pública, faz-se necessário o desenvolvimento mais acelerado de pesquisas que melhor esclareçam as relações entre o funcionamento individual e os ambientes organizacionais e comunitários.
\end{abstract}

UNITERMOS: Psicologia. Saúde Pública.

As preocupações que deram origem a esta apresentação têm, em suas raízes, uma questão fundamental: como tornar o atendimen. to psicológico mais acessível e útil a uma faixa mais ampla de nossa população, evitando que se restrinja a certos grupos limitados? A proposta de solução aqui apresen. tada é a de atuação comunitária com enfoque preventivo numa perspectiva de saúde pública.

Tendo em vista o tema deste trabalho, poder-se-ia julgar, à primeira vista, que o importante a enfatizar nesta definição seria a referência ao bem-estar mental e social. Mas, assim julgando, ignorar-se-iam algumas tendências mais recentes que atribuem, à psicologia, um papel relevante quanto à manutenção da saúde, bem como à prevenção referentes também a doenças orgânicas.

E o que nos revela, por exemplo, uma série de artigos publicados pela revista Ame- rican Psychologist. Esses artigos, embora ditados por condições vigentes nos Estados Unidos, parecem adequados à nossa realidade social, dada não só a genèralidade dos problemas que abordam, como a pertinência do enfoque de alguns no que diz respeito ao risco de saúde das populações menos favorecidas. Eles tratam da influência do comportamento e do ambiente sobre a saúde (Michael8 , 1982), empregam expressões como "saúde comportamental" e "psicologia da saúde" (Matarazzo 6 , 1982; Iscoe 5,1982 ) e encaram os problemas de saúde numa perspectiva de estreita colaboração entre psicologia e saúde pública (Michael ${ }^{8}, 1982$ e Singer e Krantz ${ }^{10}, 1982$ ), referindo-se todos à importância do esforço preventivo.

0 artigo de Michael $^{8}$ (1982), depois de afirmar "concordamos todos que chegou a hora da prevenção", fala da necessidade de uma reordenação de prioridades na área

\footnotetext{
* Trabalho apresentado no Simpósio sobre "A Psicologia Preventiva e a Realidade Brasileira", durante a $35^{\mathrm{a}}$. Reunião da Sociedade Brasileira para o Progresso da Ciência, realizada em Belém, PA, em julho de 1983.

**Do Instituto de Psicologia da Universidade de São Paulo - Cidade Universitária "Armando de Sales Oliveira" - Caixa Postal 11.454 - 05508 - São Paulo, SP.
} 
MEJIAS, N. P. O psicólogo, a saúde pública e o esforço preventivo. Rev. Saúde públ., S. Paulo, 18:155-61, 1984.

de saúde, tendo em vista quatro fatores de maior responsabilidade em termos de causa mortis: a) fatores comportamentais ou estilo de vida insalubre; b) riscos ambientais; c) fatores biológicos humanos e d) inadequações no sistema de cuidados com a saúde, atualmente em vigência. Esse Autor avalia a influência de cada um desses fatores e com base em dados de 1979, extraídos do CDC (Centre for Disease Control), conclui que $50 \%$ das mortes ocorridas nos Estados Unidos têm como fator determinante o próprio comportamento do indivíduo ou seu estilo de vida. Dos restantes 50\%, 20\% seriam devidos a fatores ambientais, $20 \%$ a fatores biológicos e apenas $10 \%$ a cuidados inadequados com a saúde. Para Michael ${ }^{8}$ parece claro que os esforços predominantes para melhorar o estado de saúde dos cidadãos americanos não se devem dirigir ao tratamento da saúde, mas aos programas de prevenção. Uma prevenção que deverá levá-los a reconhecer sua própria responsabilidade nos cuidados da saúde, concentrando-se, de um lado, em mudanças de hábitos e constumes que podem levar à doença (fumar, comer em excesso, por exemplo) e de outro, no desenvolvimento de hábitos (como a prática de exercícios físicos). Mesmo no caso de doenças específicas como a diabete, é importante considerar o tipo de vida e as modalidades de trabalho a que se dedica o diabético.

Idéias semelhantes às apresentadas por Michael $^{8}$ verificam-se no artigo de Mataraz$\mathrm{zo}^{6}$ (1982), para quem o comportamento dos indivíduos é um assunto pouco explorado no estudo e compreensão da sáude. $E$ entre os comportamentos, aparentemente mais ligados aos problemas de saúde, menciona o abuso do fumo, do álcool e do sal; a prática deficiente da higiene dental; a falha no uso de cintos de segurança nos automóveis entre outros. Aliás, segundo esse Autor, se nos últimos 80 anos os conhecimentos relacionados a moléstias infecciosas, imunologia e epidemiologia alteraram de modo marcante os padrões de doença dos americanos (reduzindo ou mesmo eliminando a tuberculose, o sarampo, a poliomielite $e$ a in- fluenza) há de se notar que ocorre, ao mesmo tempo, o aumento de casos de câncer de pulmão, de moléstias cardiovasculares, de abuso da droga e do álcool, bem como o de problemas derivados de acidentes de motocicleta e de carro. Há de se notar que estes últimos estão muitas vezes relacionados ao abuso do álcool e todos eles estão freqüentemente ligados aos comportamentos do indivíduo ou seu estilo de vida. Para Matarazzo ${ }^{6}$, o levantamento de fatos como esses confirmam palavras do médico e filósofo social Knowles (citado por Matarazzo ${ }^{6}$, 1982) para quem "mais de $99 \%$ entre nós nasce saudável e fica doente devido a uma conduta pessoal imprópria e a condiçóes am. bientais". Para ele, a solução dos problemas de saúde precária na sociedade americana envolve, primeiro, a responsabilidade individual e, segundo, a responsabilidade social, e esta última através de uma legislação adequada e de esforços voluntários privados.

Entretanto, qual teria sido a contribuição específica da psicologia quanto a essa responsabilidade?

Ainda segundo Matarazzo 6 , a psicologia não esteve alheia nessa busca de solução. Aliás, seu envolvimento foi de tal ordem, nas duas últimas décadas, que, em 1978, a "American Psychological Association" (APA) criou uma nova divisão, de número 38 , denominada psicologia da saúde.

A conceituação de psicologia da saúde adotada pela Divisão de Psicologia da Saúde, da APA, é a seguinte:

"A Psicologia de Saúde é o agregado de contribuiçōes educacionais, cientificas e pro. fissionais específicas da Psicologia à promoção e à manutenção da saúde, à prevenção e ao tratamento da doença, à identificação de correlatos etiológicos e diagnósticos da saúde $e$ da doença e respectivas disfunções. Ela visa ainda a análise e o progresso do sistema de assistência à saúde e o desenvolvimento da politica sanitária" (Matarazzo ${ }^{6}$ )

No artigo desse mesmo Autor, encontramos ainda a expressão saúde comportamental, que corresponde a um campo interdisciplinar, produto de desenvolvimentos mais recentes da medicina e da psicologia. Quanto 
MEJIAS, N. P. O psicólogo, a saúde pública e o esforço preventivo. Rev. Saúde públ., S. Paulo, 18:155-61, 1984.

ao papel da psicologia, propriamente, fala dos desafios que a saúde comportamental e a psicologia da saúde representam para a psicologia acadêmica, científica e profissional, parecendo considerar como cerne do problema a mudança de comportamento do indivíduo americano. Em outras palavras, esse Autor parece concentrar sua atenção na alteração de comportamentos relacionados a doenças, como o câncer, moléstias do coração e outras. E, para chegar a essas altera. ções, preconiza a investigação de meios para evitar o uso do fumo, do álcool, das drogas e de outras substâncias prejudiciais à saúde; $o$ uso do fio dental; o uso de cintos de segurança; exercícios regulares entre outros.

$O$ artigo de Singer e Krantz ${ }^{10}$ (1982) é de especial interesse dada sua preocupação com problemas de relações entre psicologia e saúde pública. Para esses Autores, embora as raízes do envolvimento da psicologia com questões de saúde e doença datem de mais de um século, esse envolvimento está longe de ser claro ou esclarecedor para os psicólogos. Existem pontos de contacto entre as duas áreas, mas existem, também, divergências, sobretudo no que diz respeito ao enfoque do problema. Quais seriam essas divergências?

Há de se notar que os aspectos principais da abordagem da saúde pública incluem o enfoque na prevenção primária, uma orientação no sentido do macro sistema social (a comunidade, a nação e a sociedade), intervenções e preocupações em termos de política de saúde e política social.

Já a Psicologia tende a dar, tradicionalmente, maior atenção a aspectos secundários e terciários dos problemas de saúde (e isto, talvez, com maior propriedade, se aplique à saúde mental), voltando-se primordialmente para o indivíduo. Aliás, nesse sentido, parece pertinente mencionar aqui as críticas de Saranson ${ }^{9}$ (1981) à psicologia clínica como associal e, como tal, com objetivos muito estreitos para enfrentar os conflitos da realidade social da política de saúde. $\mathrm{Pa}$ ra esse autor uma psicologia clínica sem raízes numa psicologia social realista - isto é, uma psicologia social que se considera um produto e agente cultural e histórico-social, e que se vê, por razões de lugar e status social e institucional, tanto como causa, como efeito cultural - é uma psicologia clínica mal orientada. Coerentes, pois, com o enfoque individual, os psicólogos evitam questões relativas à política de saúde, pois as consideram fora do domínio científico da psicologia. Aliás, segundo Singer e Krantz ${ }^{10}$, mesmo ao considerar os grandes grupos, o psicólogo o faz devido ao impacto que provocam sobre o comportamento individual. E para ilustrar estas considerações apresentam, como exemplo, as duas maneiras de lidar com o hábito de fumar: enquanto o psicólogo encara o problema em termos de estratégias terapêuticas para a prevenção ou extinção do hábito de fumar, o especialista em saúde pública enfatiza medidas educacionais de amplitude comunitária, levantamentos de fatores de risco epidemiológicos e mudanças na política de saúde, em ampla escala. A preocupação principal do psicólogo tem sido, portanto, o desenvolvimento de métodos para a solução de problemas quotidianos, ao invés da promoção da compreen. são científica de processos subjacentes à mudança do comportamento e promoção da saúde.

Para finalizar o exame destes artigos, parece importante salientar, ainda com base em Singer e Krantz ${ }^{10}$, as três razões principais que justificam maior consideração pelo enfoque social:

Primeiro: Existem questões cuja solução pode estar além do controle individual. É o caso, por exemplo, do acúmulo de informações mostrando que a maioria das doenças de câncer e das molésticas respiratórias estão relacionadas a condições ambientais e poluentes. E há muito já se sabe que o suicídio e assassinato estão relacionados a condições econômicas.

Segundo: as crenças e valores relacionados à saúde surgem num dado contexto social. Nossas próprias possibilidades e mesmo nossas habilidades em mudar certas crenças existem como parte de um contexto social mais amplo. 
MEJIAS, N. P. O psicólogo, a saúde pública e o esforço preventiva. Rev. Saúde públ., S. Paulo, 18:155-61, 1984.

Terceiro: Nossa responsabilidade em relação a questões de saúde ou ante a mudança de certos comportamentos não é algo individual, mas coletivo. Um exemplo desta afirmação é a mudança de enfoque no interesse da ansiedade para o stress, dentro da própria Psicologia. $\mathrm{Na}$ verdade, as duas expressões referem-se às mesmas situações, atribuindo-se, porém, determinantes internos à ansiedade e determinantes externos ao stress.

Por outro lado, o que poderia o psicólogo oferecer à saúde pública? Estes autores parecem defender o ponto de vista que a maior contribuição da psicologia é o exame dos mecanismos ligando comportamento e saúde. As características do comportamento saudável, a manutenção da saúde, as mudanças de estilo de vida são questões que podem ser abrangidas pelos princípios gerais de comportamento, que constituem o âmago da psicologia.

$O$ artigo de Iscoe 5 (1982), difere dos anteriores mudando o enfoque de interesse individual para comunitário, dando ênfase especial à psicologia da comunidade. Para ele, a psicologia da saúde deveria adotar uma perspectiva de comunidade ou saúde pública, ao invés do modelo da doença e de tratamento individual.

Além disso, esse mesmo Autor manifesta a preocupação em desenvolver uma psicologia da saúde que atinja a comunidade no seu todo. $\mathrm{E}$, nesse sentido, não como crítica, mas aviso de alerta (referindo-se, evidemente, aos Estados Unidos), afirma que as pessoas que se utilizam dos benefícios de campanhas de promoção da saúde e têm maior acesso a informações sobre saúde são geralmente aquelas pertencentes à classe média. Para ele, o insucesso dos programas de saúde mental (ainda referindo-se aos Estados Unidos, naturalmente) deve-se a uma compreensão insuficiente dos problemas do dia-a-dia e dos valores das pessoas tidas como semiqualificadas ou mesmo qualificadas. As pessoas semi-educadas ou não educadas, subempregadas ou desempregadas estão crescendo em número, diz ele, e são justamente estas as que correm o maior risco de saúde $\mathrm{e}$ as que menos se utilizam das informações contidas em campanhas de saúde. No entanto, são justamente essas as que deveriam constituir o verdadeiro alvo de uma psicologia da saúde digna de fé.

Relativamente ao aspecto de atuação, Iscoe ${ }^{5}$ salienta a necessidade de interação do psicólogo com outros profissionais da saú. de pública, acrescentando seus conhecimentos aos do grupo, de modo a intensificar os esforços em comum. Quanto às oportunidades de contribuir para a promoção da saúde, esse Autor acredita que elas se apresentam mais viáveis nas Secretaria ou Departa. mentos de Saúde, na rede escolar e em clínicas comunitárias. Mas, além disso, acredita que os psicólogos podem desempenhar um importante papel na formulação de uma política de saúde - um ponto que gostaríamos de salientar. Entretanto, preocupado ainda com os problemas de atuação do psicólogo, esse Autor chama atenção para o fato de que, embora capacitado para alterar atitudes e pomover condições para a modificação de comportamento, o psicólogo raramente apresenta o preparo e a experiência necessários para fazê-lo de uma maneira que não seja no contacto face a face, individual. A adoção do modelo de saúde pública é difícil, afirma Iscoe ${ }^{5}$, pois a prática e os procedimentos adequados à terapia individual ou de grupo podem não ser apropriados ao desenvolvimento e à aplicação de uma psicologia da saúde.

Tendo em vista a resenha apresentada e dada nossa realidade social, parece-nos de especial interesse $o$ artigo de Iscoe ${ }^{5}$, lembrando que ele propõe uma psicologia da saúde que atinja a comunidade no seu todo, abrangendo assim, as populações menos favorecidas, cada vez mais numerosas. $E$, com base no que diz esse Autor, parece adequado tentar buscar na psicologia da comunidade subsídios que mais se coadunem com a abordagem da saúde pública e seu enfoque na prevenção primária e orientação no sentido de uma política de saúde e política social. No entanto, como fazê-lo?

Antes de mais nada, é interessante verificar que, muito embora a prestação de ser- 
MEJIAS, N. P. O psicólogo, a saúde pública e o esforço preventivo. Rev. Saúde públ., S. Paulo, 18:155-61, 1984.

viços psicológicos à comunidade tenha emergido da psicologia clínica, a psicologia da comunidade procurou, desde o início, negar suas ligações com a psicologia clínica, procurando afirmar-se, não como seu subproduto, mas como uma interação de conhecimentos científicos básicos da psicologia, com ênfase na psicologia social e na psicologia do desenvolvimento (Bennett ${ }^{1}, 1965$ ).

Aliás, este é um ponto que gostaríamos de salientar: não se aplicaria uma tal concepção de psicologia da comunidade a todas as áreas de aplicação? Não constituiriam essas áreas uma interação de conhecimentos básicos, com ênfase em disciplinas diversas conforme seu enfoque?

Voltemos, porém, à psicologia da comunidade e suas relações com a psicologia clínica.

Enquanto a psicologia clínica preocupa-se com problemas individuais de ajustamento à vida, tendo como nível primordial de análise o indivíduo, a psicologia da comunidade "entrou no campo do bem-estar humano através de envolvimento direto com sistemas de organizaçấo para o controle de desvios de comportamento" e o desenvolvimento de sistemas sociais mais condizentes com as necessidades humanas (Goodstein e Sandler $^{3}, 1978$ ). E, nesse sentido, sua ênfase está na prevenção. Aliás, a psicologia da comunidade é apresentada na literatura (McClure e col. $^{7}, 1980$ ) como tendo três características principais: uma perspectiva teórica orientada para a competência e a prevenção; a preferência por uma intervenção na organização e na comunidade, em nível ecológico; e a necessidade de se fundamentar em pesquisas ecologicamente válidas.

É preciso notar, porém, que a psicologia da comunidade não ignora o indivíduo "ela apenas reconhece as relaçôes emaranhadas entre as dimensões dos sistemas sociais, os niveis de competência interpessoal e o funcionamento social" (Cowen ${ }^{2}, 1977$ ). Parece ainda importante mencionar que, muito embora o enfoque na prevenção e na competência seja uma de suas características principais ou mesmo a primordial, a psicologia da comunidade ambiciona mais do que a prevenção - seu objetivo último seria o desenvolvimento de indivíduos competentes em comunidades competentes, denominando-se comunidades competentes as que têm recursos e poder para satisfazer as suas necessidades. Quanto a estas, seriam definidas pela própria comunidade (Iscoe $\left.{ }^{4}, 1974\right)$.

Ora, lembrando que os objetivos mais amplos da Organização Mundial de Saúde é promover esforços para que todos os povos atinjam o nível mais alto possível de saúde e tendo em vista a sua definição de saúde como o bem estar físico, mental e social e não meramente a ausência de doenças, di. ríamos que o ènfoque da psicologia da comunidade está intimamente ligado ao da saúde pública.

Entretanto - e aqui chegamos ao ponto crucial de nosso tema - reconhecer as relações entre os sistemas sociais, os níveis de competência interpessoal e o funcionamento social pode ser condição necessária, mas não suficiente para compreendê-los. Assim, o avanço, os progressos da psicologia da comunidade, ou melhor, as contribuições da psicologia com enfoque na comunidade, hão de depender do desenvolvimento de pesquisas que esclareçam as relações entre o funcionamento individual e os ambientes organizacionais e comunitários. Assim, as contribuições da psicologia à saúde pública teriam como base o desenvolvimento desse tipo de pesquisa. No entanto, é justamente nesse sentido que parece haver ainda um longo caminho a percorrer.

Num levantamento realizado por McClure e col. ${ }^{7}$ (1980), sobre os tipos de estudo mais freqüentes (teórico, correlacional, quase-experimental, experimental e outros), publicados de 1973 a 1978 , verificou-se que a grande maioria dos estudos empíricos envolviam uma abordagem tradicional com enfoque em problemas a nivel individual ou de pequeno grupo. As razões para o pequeno número de pesquisas sobre intervenções a nivel comunitário, segundo esse Autor, podem ser atribuídas aos seguintes fatores: a) rejeição do modelo conceitual da psicologia da comunidade; b) falta de conhecimento e de preparo do pesquisador; c) limitações 
MEJIAS, N. P. O psicólogo, a saúde pública e o esforço preventivo. Rev. Saúde públ., S. Paulo, 18:155-61, 1984.

impostas pelo próprio contexto comunitário-institucional do pesquisador; d) a política governamental em nível municipal, estadual e federal.

Os problemas são, pois, complexos e numerosos e, na maior parte, de difícil solução. "A psicologia da comunidade anda em crise, pois os psicólogos estão questionando seus paradigmas, suas pesquisas, seu preparo e suas habilidades para aplicar seus conhecimentos à solução de problemas de comunidade", afirma Rappaport (citado por Mc. Clure e coll $^{7}, 1980$ ). Assim sendo, parece válido afirmar que as possibilidades de contribuição da psicologia à saúde pública numa perspectiva de prevenção e em âmbito comunitário estão, em última análise, vinculadas às respostas encontradas a esse questionamento. Além disso, parece interessante considerar ainda com McClure e col. ${ }^{7}$ que os psicólogos das áreas de psicologia social, psicologia clínica e, mais recentemente, psi- cologia do desenvolvimento estão procurando ampliar as perspectivas da psicologia, es. tendendo os limites do laboratório e do consultório para os domínios mais amplos da realidade social e realidade ecológica, buscando novos paradigmas, desenvolvendo novos programas de treino e preparando novas revistas científicas.

Para concluir, diríamos que as possibilidades de contribuição da psicologia à saúde pública estão condicionadas aos progressos da psicologia da comunidade e, nesse sentido, dependem de um desenvolvimento mais acelerado de pesquisas que melhor esclareçam as relações entre o funcionamento in. dividual $e$ os ambientes organizacionais $e$ comunitários. Dada, porém, a complexidade dos problemas envolvidos, acreditamos que essas pesquisas produzirão resultados mais eficazes se inseridas no âmbito de questionamento a que se refere Rappaport (citado por McClure e col. ${ }^{7}$ ).

MEJIAS, N. P. [The psychologist in public health - efforts at prevention]. Rev. Saúde públ., S. Paulo, 18: $155-61,1984$

ABSTRACT: Focusing on the contributions of psychology to prevention in Public Health, this paper presents a review of several articles concerning the health behavior of individuals and the relationships between environmental conditions, behavior and health. On the other hand, taking into consideration points of contact and divergence between clinical psychology and Public Health, this review suggests that community psychology should be the area of psychology whose objectives are closer to those of the World Health Organization - a suggestion based on their common concern with the relationships between organizational systems and individual behavior, and the development of social systems congruent with human needs. Still, given the scarcity of available empirical data, this paper ends with the conclusion that a more effective contribution to Public Health would be attained if a greater number of research projects were developed clarifying the relationships between individual behavior and organizational and community environments.

UNITERMS: Psychology. Public health. Community health services.

\section{REFERENCIAS BIBLIOGRÁFICAS}

1. BENNETT, C. Community psychology: impressions of the Boston Conference on the Education of Psychologists for Community Mental Health. Amer. Psychol., 20: 102-7, 1965 .

2. COWEN, E. L. Baby - steps toward primary prevention. Amer. Community Psychol., 5: 1-22, 1977.
3. GOODSTEIN, L. D. \& SANDLER, I. Using psychology to promote human welfare: a conceptual analysis of the role of community psychology. Amer. Psychol, 33: 88392,1978 .

4. ISCOE, I. Community psychology and the competent community. Amer. Psychol., 29: $607-13,1974$. 
MEJIAS, N. P. O psicólogo, a saúde pública e o esforço preventivo. Rev. Saúde púbL, S. Paulo, 18:155.61, 1984.

5. ISCOE, I. Toward a viable community health psychology: Caveats from experiences of the community mental health movement. Amer. Psychol., 37: 961-5, 1982.

6. MATARAZZO, J. D. Behavior health's challenge to academic, scientific and professional pyschology. Amer. Psychol., 37: 1-14, 1982.

7. McCLURE, L.; CANNON, D.; BELTON, E. ; D'ASCOLI, C.; SULLIVAN, B.; ALLEN, S.; CONNOR, P.; STONE, P. \& McCLURE, G. Community psychology concepts and research base: promise and product. Amer. Psychol., 35: 1000-11, 1980.
8. MICHAEL, J. M. The second revolution in health: health promotion and its environmental base. Amer. Psychol, 37: 936-42, 1982.

9. SARANSON, S. B. An asocial psychology and a misdirected clinical psychology. Amer. Psychol., 36: 827-36, 1981.

10.SINGER, J. E. \& KRANTZ, D. S. Perspectives on the interface between psychology and public health. Amer. Psychol., 37: 955-60, 1982.

Recebido para publicação em 25/10/1983. Aprovado para publicaçāo em 19/12/1983. 